\title{
A comparative review of the list of essential medicines of three Indian states: Findings and implications
}

\section{Venkatesh Narayan, Maulik Chokshi', Habib Hasan'}

International Clinical Epidemiology Network, ${ }^{1}$ Indian Institute of Public Health, New Delhi, India

Address for the Correspondence: Dr Venkatesh Narayan, Flat No. 351, Sector A,

Pocket C, Vasant Kunj, New Delhi - 110 070, India. E-mail: drvenkateshnarayan@ gmail.com

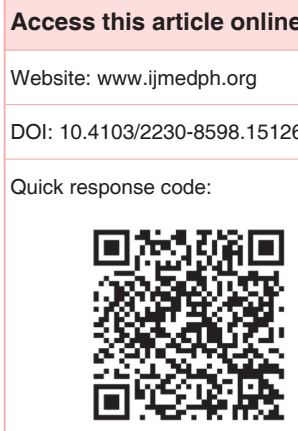

Introduction: Essential medicines lists are a key instrument for improving quality and equitable access to health care. The National List of Essential Medicines of India 2011 is modeled on the WHO Essential Medicines List and Indian states (adopting the National List) are free to include other medicines as needed. Materials and Methods: National List of Essential Medicines of India 2011 contains a total of 287 medicines for provision at primary and secondary level of health facilities. The International Nonproprietary Name of these medicines was compared with the List of Essential Medicines (LEM) of three states, that is, Bihar, Rajasthan and Tamil Nadu for the inclusion patterns. Results: A large number of medicines from the National List of Essential Medicine (NLEM) were missing from the state LEM's, especially Bihar. The sections on Anticonvulsants, Diuretics, Psychotherapeutic, Antiallergics and Oxytocics were comprehensively included by both Rajasthan and Tamil Nadu. Furthermore, the analgesic and anti-infective medicines were largely included. However, the sections of antidotes, diagnostic agents and ophthalmological preparations were grossly deficient. Similarities were found across states in their patterns of both inclusion and exclusion of medicines. Conclusion: The analysis reveals that the extent of inclusion of NLEM medicines in state LEM's is deficient with variable patterns across states. This in turn has implications for drug availability, prescription patterns, and rational drug use. As some drugs are repeatedly there in NLEM but not included by states, a discussion based consultative approach for better coherence across the lists could lead to further optimization and utilization of LEM's which would aid in improved access to medicines.

Key words: Essential medicines, inclusion of medicines, India essential medicine list

\section{INTRODUCTION}

WHO defines Essential medicines as those that satisfy the health care needs of the majority of the population and are selected with due regard to public health relevance, evidence on efficacy and safety, and comparative cost-effectiveness. ${ }^{[1]}$ Availability of a limited list of carefully selected essential medicines within the context of functioning health systems is among the key instruments to improve quality and equitable access to health care. ${ }^{[2]}$ WHO published the first WHO Model list of essential medicines (EML) in 1977 thereby providing a template which could help countries prepare their own lists $^{[3]}$ as also emulated by the $17^{\text {th }}$ WHO model EML 2011. ${ }^{[4]}$ The Indian National List of Essential Medicine (NLEM) preface states that it is modeled on the WHO EML. India's first NLEM was prepared and released in 1996 which got revised in 2003 and the current 2011 version is its $3^{\text {rd }}$ revision. ${ }^{[5]}$

Within the recent debates over Universal Health Coverage in India, the issue of access to medicines has received significant attention. ${ }^{[6]}$ State schemes of free access to medicines as demonstrated by Rajasthan ${ }^{[7]}$ and Tamil $\mathrm{Nadu}^{[8]}$ have paved the path for universal access to medicines. Several Indian states have developed their state LEM's. Often, the state-level technical advisory committees study the WHO list, NLEM and also the disease pattern prevalent in the state to derive state LEM. ${ }^{[9]}$ States adopting the National List are free to include other medicines as considered adequate to treat additional diseases of local importance. While comparative analysis of LEM's has been carried out by few earlier studies, often, the focus is on specific sections ${ }^{[10,11]}$ (such as Reproductive Health, Maternal and Child health) or an overall 
comparison of LEM's of countries against the WHO lists. ${ }^{[12]}$ Health being a state subject in India, adequate focus needs to be devoted to the state LEM's for improving the drug availability at health institutions. As Government of India takes hesitant steps toward universal access to medicines by asking states to review and adopt NLEM as per state requirements, ${ }^{[6]}$ this review attempts to aid the process. Since earlier studies have not specifically addressed the state LEM's, this study hopes to contribute to the knowledge in this arena.

\section{MATERIALS AND METHODS}

The study aims to compare the inclusion of the medicines listed in the NLEM across three states. The methods were adapted from earlier studies ${ }^{[10,11]}$ on the subject and the methodology was suitably modified to compare India's LEM with state LEM's. The state selection was carried out purposively. Rajasthan has recently launched a recent free medicines scheme ${ }^{[7]}$ and was thus selected to see if their LEM offered an adequate inclusion of medicines. The state of Tamil Nadu is well known for its successful health initiatives including its Tamil Nadu Medical Services Corporation (TNMSC) model ${ }^{[8]}$ and was thus included. To also review a state with comparatively poorer health indicators and performance, ${ }^{[13]}$ Bihar LEM was chosen to assess its inclusion. Rajasthan Medical Services Corporation maintains a publically available collection of selected essential medicines lists at http:/ / www.rmsc.nic. in/Drug_Procurement.html which contained lists from four states (Rajasthan, ${ }^{[14]} \mathrm{MP}$, Bihar, ${ }^{[15]} \mathrm{TN}^{[16]}$ ) at the time this study was conducted. For this study, the essential medicines lists for the requisite states were then selected, resulting in three unique state lists for analysis. These were then compared from NLEM available at drug regulatory authority of India, the Central Drugs Standard Control Organization (http:/ /www. cdsco.nic.in). The lists were downloaded and reviewed by comparing them with each other. Each LEM was evaluated for concordance with the medicines listed in the NLEM by the lead authors. Any discrepancies were adjudicated by the third author.

National List of Essential Medicine 2011 has categorized medicines according to therapeutic area. Thus certain medicines appear in $>1$ category. The list comprises a total of 348 medicines (excluding repetitions), which also includes all the medicines being provided under various National Health Programs. From the total of 348 medicines in NLEM, 181 medicines have been categorized for health institutions at all levels (P-primary, S-secondary, T-tertiary) whereas 106 medicines have been listed for institutions at secondary and tertiary (S, T) levels. The remaining 61 drugs are categorized only for tertiary level health institutions and more than half of these comprise of anticancer drugs. This analysis being more focused on drug inclusions and access at primary and secondary level, the tertiary category and its 61 medicines were excluded from the comparative analysis. Thus, from these 348 medicines listed in NLEM 2011, a medicines list comprising a total of 287 (181 P, S, T + $106 \mathrm{~S}, \mathrm{~T})$ medicines up to secondary level health institutions was derived.

The comparison was carried out on the basis of the International Nonproprietary Name (INN) of these 287 medicines and the criterion for noninclusion was the absence of NLEM medicines INN in the state LEM. Medicines are additionally characterized by their strength, dosage form (e.g., syrup, cream, injection) and fixed dose combinations (FDC's). However, the alphabetical list comprising of all the 348 NLEM medicines has listed only the INN of the medicines. Thus, for the purpose of comparison, listing of the INN in the state list was considered as inclusion irrespective of the strength or dosage forms. Listing of the INN, even within FDC was considered as the inclusion in the state LEM. For example, ferrous sulfate combination with folic acid was taken as inclusion of both of these chemical entities. The drugs under National Health Programs (e.g., Tuberculosis, Malaria, HIV) are often supplied through separate programs under central support and thus were considered to be included for state lists. Thus, the noninclusions derived from this analysis are conservative estimates, allowing for the above considerations, barring which the extent of noninclusions would be even larger.

\section{RESULTS}

\section{Comparison of Bihar list of Essential Medicines 2009 with National list of Essential Medicine}

The Bihar LEM 2009 lists the drugs alphabetically for each institution both for outdoor and indoor. The Outdoor list for District Hospital that corresponds with secondary level of health facility comprises of total 41 medicines that also includes Plaster of Paris. It also includes four FDC's (e.g., Dicyclomine with Paracetamol). It comprises of certain ambiguities such as simply writing "cough syrup/sedative" without specifying the actual contents and listing of the medicine Rabeprazole as alternative to Ranitidine. The Indoor list comprises a total of 193 medicines that also includes items such as dressing and sutures. Typological errors such as atropine being written twice and spelling mistakes exist in the list. Furthermore, antibiotics such as Amoxiclav and Vancomycin which have been listed by NLEM at tertiary level are listed at District Hospital. Against the 287 medicines listed in NLEM for primary and secondary level, a total of 182 medicines were found to be present in the Bihar LEM with the rest 105 medicines not being included [Table 1].

\section{Comparison of Rajasthan and Tamil Nadu list of Essential Medicines (2012) with National list of Essential Medicine}

The Rajasthan LEM listed a total of 477 medicines and also the category (Primary, Secondary, Tertiary) of health institution for its

$\begin{aligned} & \text { Table 1: Comparison of Bihar, Rajasthan } \\
& \text { and Tamil Nadu LEM with NLEM }\end{aligned}$
\begin{tabular}{lccc} 
Bihar & Rajasthan \\
LEM (\%) & Tamil Nadu $(\%)$ & LEM (\%) \\
\hline $\begin{array}{l}\text { Included medicines (out } \\
\text { of } 287 \text { in NLEM) }\end{array}$ & $182(63.4)$ & $236(82.2)$ & $207(72.7)$ \\
$\begin{array}{l}\text { Nonincluded medicines } \\
\text { (out of } 287 \text { in NLEM) }\end{array}$ & $105(36.6)$ & $51(17.8)$ & $78(27.3)$ \\
\hline
\end{tabular}

NLEM = National list of essential medicine, $\mathrm{LEM}=$ List of essential medicine 
availability. The Tamil Nadu LEM listed a total of 260 medicines with their strengths, but without the category of health institutions. As evident from Table 1, of the 287 medicines listed in NLEM up to secondary level, $236(82.2 \%)$ have been included by the Rajasthan LEM as against $207(72.7 \%)$ in Tamil Nadu LEM. Thus, as compared to the NLEM, 51 and 78 drugs are not included by Rajasthan and Tamil Nadu respectively.

Utilizing the availability of updated 2012 therapeutic area wise LEM's of both states, comparisons across sections were carried out to look further into these inclusions. The section-wise detailed analysis for the five most included sections [Figure 1] revealed identical patterns for both states. For Section 5 of Anticonvulsants/Antiepileptics, Section 16 of Diuretics, and Section 24 of Psychotherapeutic Medicines comprising of six, four and eight medicines respectively, all drugs listed in NLEM were also included in the state LEM's. For Section 3 of Antiallergics and Medicines used in Anaphylaxis and Section 22 of Oxytocics and Antioxytocics, which comprised of nine and five medicines respectively, only Dexchlorpheniramine Maleate and Terbutaline were missing in corresponding therapeutic category of both states.

Section 2 of NLEM has two subsections. The Analgesics and Antipyretics (nonsteroidal antiinflammatory drugs, Opioids) had total six medicines of which while Rajasthan had all, Tramadol had not been included by Tamil Nadu [Table 2]. The total medicines for Gout and Rheumatoid Disorders listed in NLEM were seven,

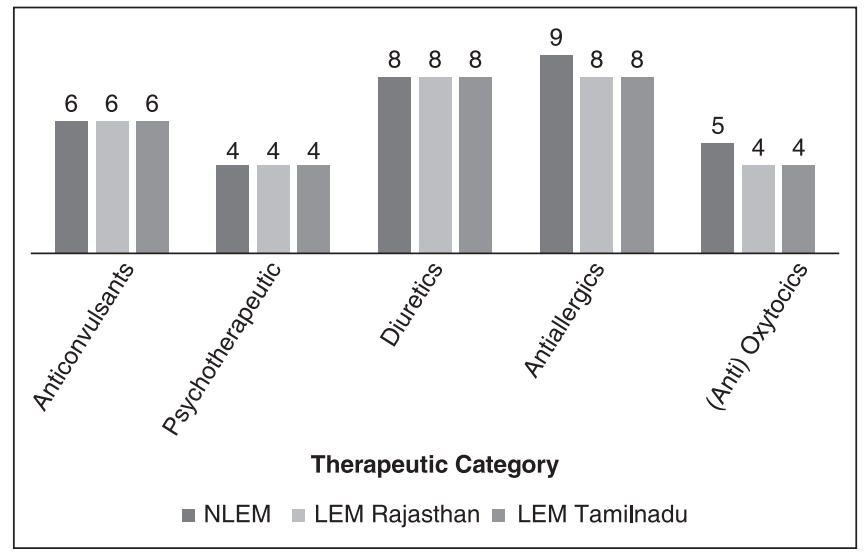

Figure 1: Most included sections from National List of Essential Medicine in List of Essential Medicines of Rajasthan and Tamil Nadu of which both Rajasthan and Tamil Nadu had included two each. While the NLEM has listed Diclofenac as medicine only for tertiary level health institutions, Rajasthan LEM includes FDC's of Diclofenac and Ibuprofen with Paracetamol even at Primary Health Centers. Section 6 of NLEM comprises of a total of 63 Antiinfective Medicines. While Rajasthan had included 58 of them, 55 anti-infectives had also been listed by Tamil Nadu.

While assessing the therapeutic categories which had the most number of excluded medicines [Figure 2], while the NLEM consisted of 13 antidotes and other substances used in poisonings, only six and four of these had been included by Rajasthan and Tamil Nadu respectively. The most deficient section was that of diagnostic agents comprising a total of 11 ophthalmic and radiocontrast agents of which while Rajasthan had included five medicines, Tamil Nadu included only one. Similarly, another deficient category was section 21 of NLEM comprising of 15 Ophthalmological Preparations of which Tamil Nadu had included six preparations whereas Rajasthan had most (11) of them.

\section{Similarities across Rajasthan and Tamil Nadu list of Essential Medicine's}

As states are showing similar and related patterns of inclusion and noninclusion, Table 3 was derived comprising of the five most included sections by Rajasthan and Tamil Nadu along with the cardiac, gastrointestinal and respiratory medicines w.r.t. the

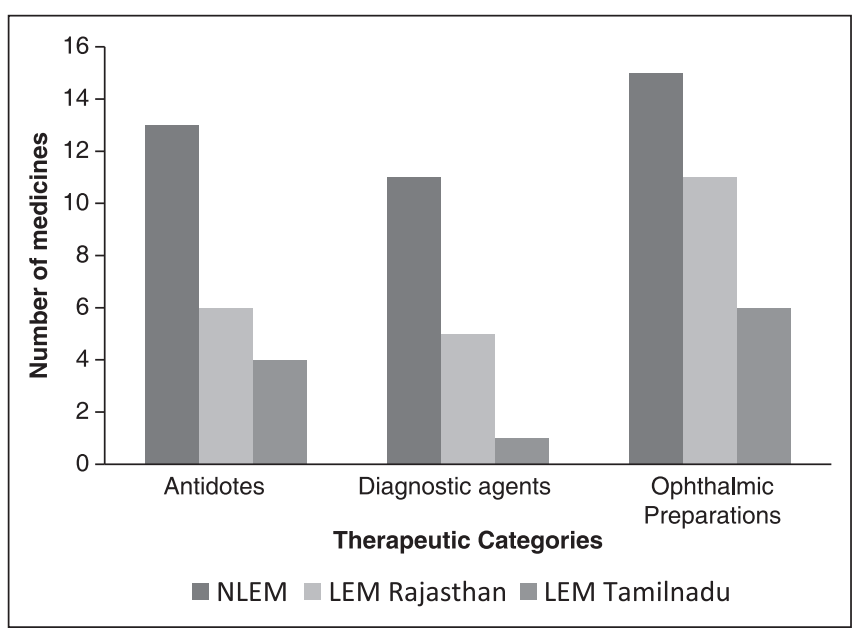

Figure 2: Most nonincluded sections from National List of Essential Medicine in List of Essential Medicines of Rajasthan and Tamil Nadu
Table 2: Analgesics and antiinfectives

\begin{tabular}{|c|c|c|c|c|}
\hline Therapeutic category & NLEM & $\begin{array}{c}\text { LEM } \\
\text { Rajasthan }\end{array}$ & $\begin{array}{l}\text { LEM Tamil } \\
\text { Nadu }\end{array}$ & Nonincluded medicines \\
\hline Section: 2 - Analgesics and antipyretics (NSAID's, opioids) & 6 & 6 & 5 & Tramadol \\
\hline Medicines for gout and rheumatoid disorders & 7 & 2 & 2 & $\begin{array}{l}\text { Allopurinol, colchicine, sulfasalazine, } \\
\text { hydroxychloroquine phosphate, leflunomide }\end{array}$ \\
\hline $\begin{array}{l}\text { Section: } 6 \text { - Anti-infective medicines (also includes the } \\
\text { medicines under National Health Programmes for leprosy, } \\
\text { tuberculosis, HIV, KalaAzar and Malaria) }\end{array}$ & 63 & 58 & 55 & $\begin{array}{l}\text { Piperazine, praziquantel, sulphadiazine, } \\
\text { nystatin, diloxanide furoate }\end{array}$ \\
\hline Total & 76 & 66 & 62 & \\
\hline
\end{tabular}

NLEM = National list of essential medicine, LEM = List of essential medicine, NSAID's = Nonsteroidal anti-inflammatory drugs 


\begin{tabular}{|c|c|c|c|c|}
\hline Therapeutic category & NLEM & $\begin{array}{c}\text { LEM } \\
\text { Rajasthan }\end{array}$ & $\begin{array}{l}\text { LEM Tamil } \\
\text { Nadu }\end{array}$ & Nonincluded medicines (only Tamil Nadu*) \\
\hline Section: 7 - Antimigraine medicines & 4 & 3 & 3 & Dihydro ergotamine \\
\hline Section: 9 - Antiparkinsonism medicines & 3 & 2 & 2 & Bromocriptine \\
\hline Section: $10-$ Medicines affecting blood & 9 & 6 & 6 & Iron dextran, protamine sulphate, warfarin \\
\hline Section: 15 - Disinfectants (4) and antiseptics (8) & 12 & 10 & 9 & $\begin{array}{l}\text { Acriflavin }+ \text { glycerin, chlorhexidine }{ }^{*} \text {, potassium } \\
\text { permanganate }\end{array}$ \\
\hline $\begin{array}{l}\text { Section: } 20-\text { Muscle relaxants and cholinesterase } \\
\text { inhibitors }\end{array}$ & 5 & 4 & 3 & Pyridostigmine, vecuronium* \\
\hline Section: 12 - Cardiac medicines & 24 & 22 & 21 & Metoprolol, adenosine, losartan* \\
\hline Section: $17-$ Gastrointestinal medicines & 15 & 13 & 10 & Famotidine*, ondansetron*, 5-ASA, hyoscine*, ispaghula \\
\hline $\begin{array}{l}\text { Section: } 25-\text { Medicines acting on the respiratory } \\
\text { tract }\end{array}$ & 6 & 5 & 2 & $\begin{array}{l}\text { Beclomethasone dipropionate*, ipratropium*, } \\
\text { iextromethorphan*, codeine phosphate }\end{array}$ \\
\hline Total & 78 & 65 & 56 & \\
\hline
\end{tabular}

NLEM = National list of essential medicine, LEM = List of essential medicine

NLEM. The nonincluded medicines were similar across both states and comprised of medicines such as Dihydro ergotamine, Bromocriptine, Iron dextran and Warfarin.

Also, Section 12 of NLEM that listed a total of 24 Cardiac medicines of which both Rajasthan and Tamil Nadu had not included Metoprolol and adenosine. Section 17 of gastrointestinal medicines had total of 15 medicines in NLEM. Rajasthan had included most of them (13 out of 15) and ten drugs were included by Tamil Nadu. However, section 25 for Medicines acting on the respiratory tract provides a different pattern. While the NLEM has six medicines of which five are included by Rajasthan, only two have been listed by Tamil Nadu.

\section{DISCUSSION}

The results above point out specific issues, the most remarkable being the limited overall number of medicines included in state LEM's as compared to NLEM. Noninclusion of 105 drugs in Bihar LEM shows incorporation of the limited number of drugs when compared against NLEM and also the LEM of Rajasthan and Tamil Nadu pointing out its relative inadequacy [Table 1]. In contrast to this, the states of Rajasthan and Tamil Nadu has included 236 $(82.2 \%)$ and 207 (72.7\%) medicines in their respective state LEM's. The finding corroborates with the success of the free medicines scheme and TNMSC model in the states of Rajasthan ${ }^{[7]}$ and Tamil $\mathrm{Nadu}^{[8]}$ respectively. Figure 1 also shows their comprehensive inclusion patterns as evident from the comparison of the most included sections.

\section{Nonsteroidal antiinflammatory drugs and antiinfectives}

The section on [Table 2] Analgesics and Antipyretics having six drugs in NLEM are comprehensively included by Rajasthan (all six) while Tamil Nadu has excluded only Tramadol. This is explained by earlier studies which show that analgesics are consistently among the most commonly prescribed drugs. ${ }^{[13,17]}$ Also, though the NLEM includes Diclofenac only at tertiary level, the FDC's of Diclofenac and Ibuprofen with Paracetamol are included from primary level onwards by Rajasthan. While FDC's are to be avoided in LEM's, their inclusion probably reflects patient demand and also doctor's preference for these analgesics.

About the antiinfectives, interestingly [Table 2] most from NLEM are present in LEM's of both Rajasthan (58 of 63) and Tamil Nadu (55 of 63). LEM's are a necessary step for controlling antibiotic misuse and thus preventing drug resistance. ${ }^{[18]}$ However, an earlier study in Delhi shows that though certain higher antibiotics are provided only for restricted usage, they get prescribed frequently probably due to doctor's preference of or the unavailability of alternative antibiotics. ${ }^{[19]}$ Studies have pointed out the nonjudicious selection due to lack of knowledge, time or the need to finish the near expiry drugs. ${ }^{[13,20]}$ It has been demonstrated that in addition to financial considerations, perceived demand and expectation from the patients has also promoted antibiotic overuse. ${ }^{[20]}$

\section{Exclusion patterns}

The possible reasons for noninclusion could be multi-fold as it is possible that certain drugs in NLEM may be expensive and thus not included. Certain drugs are consistently missing across states reflecting that they are either unnecessarily included in NLEM, that is, inclusion could have been avoided or the prevailing disease and prescription patterns in the state does not require these drugs. This is seen [Table 2] for the medicines for Gout and Rheumatoid Disorders (total seven), of which both Rajasthan and Tamil Nadu included two, that is, majority were not considered as fit for inclusion by the states.

Some exclusions are explained on pharmacological basis. The noninclusion of the antiallergic Dexchlorpheniramine Maleate by both Rajasthan and Tamil Nadu can be explained for by the simultaneous presence of Chlorpheniramine Maleate as also pointed out by Manikandan and Gitanjali. ${ }^{[21]}$ Also, drugs with limited usage like ether ${ }^{[21]}$ would have been intentionally excluded by states. These explanations are also supported by a study done by Delhi Society for Promotion of Rational Use of Drugs (DSPRUD) which showed that from 16 states, 14 states used criteria such as safety, efficacy and cost while selecting the medicines for LEM..$^{[9]}$ Also, as the LEM's get updated at a gap of few years, the changes in NLEM 
may reflect in state LEM after a certain period based on evaluation by the state committee.

Since states frequently follow the pattern of WHO EML while preparing their own $\mathrm{LEM},{ }^{[9]}$ it can also explain some exclusions by states. For the specific categories of radio contrast and ophthalmic preparations, earlier reviews have expressed dissatisfaction over the NLEM including six ophthalmic antiinfectives and eight radio contrast media whereas the WHO lists only three medicines against each of these categories. ${ }^{[21,22]}$ While this probably also reflects the growing dependence on diagnostics, utilization of items such as diagnostic reagents is often constrained by absence of the requisite equipment's or personnel at the health institutions. ${ }^{[23]}$ Also, there could be local procurement for certain items and higher drugs, especially at specific specialty centers or blood banks but even then, they should be listed in the state LEM.

\section{Similarities across states}

Similarities across states in patterns of inclusion and exclusion are seen for several categories [Table 3]. This therapeutic category wise analysis also points out possible reasons for noninclusion of certain medicines. As depicted in Table 3, the five sections of maximum drug inclusions by both Rajasthan and Tamil Nadu have related patterns of exclusion. It is noteworthy that provision of drugs such as Warfarin (not included by both states) and heparin, which need intensive monitoring may not be feasible at ill equipped health institutions. For Gastrointestinal medicines, the excluded drugs include medicines such as Famotidine and Ondansetron. An earlier review has questioned the inclusion of Famotidine in NLEM when Omeprazole and Ranitidine are already on the list. ${ }^{[21]}$ The inclusion of additional medicines of same class without any major advantages in efficacy and safety clearly violates the selection principles of LEM. The similarity across state LEM's has also been discussed in a DSPRUD study where several states mentioned consulting LEM's of other states while preparing their own LEM. ${ }^{[0]}$ They observed that in many state LEM's, the drugs included were similar with a minor difference in certain groups of drugs.

\section{Other issues}

Though the therapeutic category wise analysis couldn't be carried out for Bihar, its LEM presents with several issues for consideration. The inclusion of irrational FDC's such as a combination of Dicyclomine with Paracetamol is entirely in violation of principles of LEM. The listing of nonspecific medicines such as simply writing "cough syrup/sedative" without specifying the actual contents and providing the nonessential medicine Rabeprazole as an alternative to the essential drug Ranitidine defeats the whole purpose of LEM preparation. Another important aspect is of tertiary level antibiotics such as Amoxiclav and Vancomycin being listed at secondary level health institutions as such discrepancy has implications for drug resistance. ${ }^{[18]}$ These inconsistencies are augmented by typological errors of repetitions (atropine listed twice) and simultaneous inclusion of surgical items such as dressing, Plaster of Paris and sutures. It needs reemphasis that these being reference documents, such errors are absolutely undesirable as also pointed out earlier for NLEM ${ }^{[21,24]}$ which also includes spelling mistakes and incorrect statistics. Also, in DSPRUD study, only seven states mentioned consulting the NLEM while preparing the state LEM. ${ }^{[9]}$ Rather, in some states the Health Department was not even aware of the NLEM. This reflects a lack of utilization and awareness coupled with limited advocacy and dissemination of NLEM at that time.

\section{Implications}

Mistakes in LEM in turn have multiple consequences. It influences the prescription patterns, treatment guidelines, national health programs, rational use of medicines and has far reaching consequences in addition to the economic and logistic implications. LEM being a tool that often gets utilized for procurement of medicines and the medicines in the list become a standard, ${ }^{[25]}$ this at times influences the manufacturing of pharmaceutical companies due to assured demand. In several states, the LEM's are prepared based on NLEM ${ }^{[9]}$ thereby incorporating and further propagating its mistakes. These issues with LEM's often don't get rectified probably because the LEM's are not being utilized or implemented for their mandated purpose. The lack of reliable data and evidence for disease prescription and utilization patterns might be responsible for nonrequisite medicines to enter the LEM's, either from unrealistic WHO standards ${ }^{[24]}$ or other vested interests including industry influence.

The study findings are constrained by its methodological limitations. Essential drug selection is a state-specific process with decisions made upon consideration of several factors including budgetary allocations, disease prevalence, drug prescription and utilization patterns and population morbidity profile. Though the study does propose these as the probable reasons behind exclusion of certain drugs, it doesn't carry out an state specific analysis of these factors and thus provides a limited understanding of the actual reasons behind these decisions. This however would require a further in-depth study comprising of analysis of state specific processes including field visits and interviews with key people which lies outside the scope of this particular study and could be taken up as a separate study altogether.

Earlier studies have shown the differential extents of adoption of WHO list across countries. ${ }^{[10-12]}$ This study takes the analysis to the state level adding to the previous studies on $\operatorname{NLEM}^{[0,21]}$ and thereby contributing to the understanding on adoption of National list across the states. With the increased emphasis on utilizing the NLEM for state LEM's, ${ }^{[6]}$ these findings assume greater importance. Though the study could provide conservative estimates only due to the methodological considerations, it still provides valuable insights into the inclusion patterns of which the underlying rationale could be explored by further studies into the issue.

\section{CONCLUSION}

This analysis reveals that the extent of inclusion of NLEM medicines in state lists is variable that influences the resultant processes towards 
access to medicines. Such NLEM comparisons with state LEM could be utilized to improve the comprehensiveness of State LEM's and vice versa which shall serve as an important step in optimizing the utilization of LEM's. With some drugs being repeatedly there in NLEM but not included by states, a discussion based consultative approach for better coherence across the lists could yield better outcomes. Also, while the analysis presents us with several inclusion and noninclusion patterns of certain sections and medicines, future research needs to explore the rationale underlying these patterns.

\section{REFERENCES}

1. World Health Organization. The Selection of Essential Medicines. WHO Policy Perspectives on Medicines. Geneva: World Health Organization; 2002.

2. World Health Organization. WHO Policy Perspectives on Medicines Equitable Access to Essential Medicines: A Framework for Collective Action. Geneva: World Health Organization; 2004.

3. World Health Organization. Medicines Use in Primary Care in Developing and Transitional Countries: Fact Book Summarizing Results from Studies Reported Between 1990 and 2006. Geneva: World Health Organization; 2009. Available from: http://www.who.int/medicines/publications/primary_ care_8April09.pdf. [Last accessed on 2013 Oct 20].

4. WHO Model List of Essential Medicines. Available from: http://www.who. int/medicines/publications/essentialmedicines/en/index.html. [Last cited on 2013 Oct 20; Last accessed on 2011 Mar 17].

5. National List of Essential Medicines of India, 2011. Available from: http:// www.cdsco.nic.in/. [Last accessed on 2013 Oct 20].

6. Bhaumik S, Biswas T. Free medicine for all in India. CMAJ 2012;184: E783-4.

7. Lodha SL. Free medicines and free diagnosis: A step towards social security measures by Rajasthan State in India. Public Policy Adm Res 2013;3:41-7.

8. Revikumar KG, Veena R, Lekshmi S, Manna PK, Mohanta GP. Tamil Nadu Medical Services Corporation - A critical study on its functioning during the period 1995-2012. Int J Pharma Chem Sci 2013;2:1691-705.

9. Report on Analysis and Comparison of Essential Medicines Lists in Public Sector in Different States of India. Delhi: Delhi Society for Promotion of Rational Use of Drugs.

10. PATH, The World Health Organization, and the United Nations Population Fund. Essential Medicines for Reproductive Health: Guiding Principles for Their Inclusion on National Medicines Lists. Seattle: PATH; 2006. Available from: http://www.who.int/reproductivehealth/publications/ general/a91388/en/. [Last accessed on 2013 Mar 10].
11. Hill S, Yang A, Bero L. Priority medicines for maternal and child health: A global survey of national essential medicines lists. PLoS One 2012;7:e38055.

12. Laing R, Waning B, Gray A, Ford N, 't Hoen E. 25 years of the WHO essential medicines lists: Progress and challenges. Lancet 2003;361: 1723-9.

13. Selvaraj S, Chokshi M, Hasan H, Kumar P. Improving Governance and Accountability in India's Medicine Supply System. Draft Report Submitted to Results for Development Institute. New Delhi: Public Health Foundation of India; 2010.

14. Rajasthan Essential Medicine List; 2012. Available from: http://www.rmsc nic.in/pdf/EDL\%20Raj-2012.pdf. [Last accessed on 2013 Mar 10].

15. Bihar Essential Medicine List; 2009. Available from: http://www.rmsc.nic. in/pdf/ELD-Bihar.pdf. [Last accessed on 2013 Mar 10].

16. Tamil Nadu Essential Medicine List; 2012. Available from: http://www rmsc.nic.in/pdf/EDL-Tamilnadu.pdf. [Last accessed on 2013 Mar 10]

17. Bapna JS, Tekur U, Gitanjali B, Shashindran $\mathrm{CH}$, Pradhan SC, Thulasimani $\mathrm{M}$, et al. Drug utilization at primary health care level in southern India. Eur J Clin Pharmacol 1992;43:413-5.

18. Ganguly NK, Arora NK, Chandy SJ, Fairoze MN, Gill JP, Gupta U, et al. Rationalizing antibiotic use to limit antibiotic resistance in India. Indian J Med Res 2011;134:281-94.

19. Kotwani A, Holloway K. Trends in antibiotic use among outpatients in New Delhi, India. BMC Infect Dis 2011:11:99.

20. Kotwani A, Wattal C, Katewa S, Joshi PC, Holloway K. Factors influencing primary care physicians to prescribe antibiotics in Delhi India. Fam Pract 2010;27:684-90.

21. Manikandan S, Gitanjali B. National list of essential medicines of India: The way forward. J Postgrad Med 2012;58:68-72.

22. Prakash VB. A critical look at the ophthalmological preparations in the national list of essential medicines of India 2011. J Postgrad Med 2013;59:159-60.

23. Gill K. A Primary Evaluation of Service Delivery Under the National Rural Health Mission (NRHM): Findings from a study in Andhra Pradesh, Uttar Pradesh, Bihar and Rajasthan. Delhi: Planning Commission of India, Government of India; 2009.

24. Gitanjali B. The national essential medicines list of India: Time to revise and purge the mistakes. J Pharmacol Pharmacother 2010;1:73-4.

25. Chirac P. Translating the essential drugs concept into the context of the year 2000. Trans R Soc Trop Med Hyg 2003;97:10-2.

How to cite this article: Narayan V, Chokshi M, Hasan H. A comparative review of the list of essential medicines of three Indian states: Findings and implications. Int $\mathrm{J}$ Med Public Health 2015;5:71-6.

Source of Support: Nil, Conflict of Interest: None declared. 